\title{
Influence of ApoE Genotype and Clock T3111C Interaction with Cardiovascular Risk Factors on the Progression to Alzheimer's Disease in Subjective Cognitive Decline and Mild Cognitive Impairment Patients
}

\author{
Valentina Bessi ${ }^{1, *}\left(\mathbb{D}\right.$, Juri Balestrini ${ }^{1}$, Silvia Bagnoli ${ }^{1}$, Salvatore Mazzeo ${ }^{1}$, Giulia Giacomucci ${ }^{1}(0$, \\ Sonia Padiglioni ${ }^{1}$, Irene Piaceri ${ }^{1}$, Marco Carraro ${ }^{1}$, Camilla Ferrari ${ }^{1}$, Laura Bracco ${ }^{1}$, \\ Sandro Sorbi ${ }^{1,2}$ and Benedetta Nacmias ${ }^{1}$ \\ 1 Department of Neuroscience, Psychology, Drug Research and Child Health-University of Florence-Viale \\ Pieraccini 6, 50139 Florence, Italy; juri.balestrini@gmail.com (J.B.); silvia.bagnoli@unifi.it (S.B.); \\ salvatore.mazzeo@unifi.it (S.M.); giuliagiacomucci.md@gmail.com (G.G.); sonia_padiglioni@libero.it (S.P.); \\ irene.piaceri@gmail.com (I.P.); marco.carraro@unifi.it (M.C.); camilla.ferrari@unifi.it (C.F.); \\ bracco.neuro@gmail.com (L.B.); sandro.sorbi@unifi.it (S.S.); benedetta.nacmias@unifi.it (B.N.) \\ 2 IRCCS Fondazione Don Carlo Gnocchi, via di Scandicci 269, 50143 Florence, Italy \\ * Correspondence: valentina.bessi@unifi.it; Tel.: +39-05-7948660; Fax: +39-05-7947484
}

Received: 11 May 2020; Accepted: 27 May 2020; Published: 29 May 2020

check for updates

\begin{abstract}
Background: Some genes could interact with cardiovascular risk factors in the development of Alzheimer's disease. We aimed to evaluate the interaction between ApoE $\varepsilon 4$ status, Clock T3111C and Per2 C111G polymorphisms with cardiovascular profile in Subjective Cognitive Decline (SCD) and Mild Cognitive Impairment (MCI). Methods: We included 68 patients who underwent clinical evaluation; neuropsychological assessment; ApoE, Clock and Per2 genotyping at baseline; and neuropsychological follow-up every 12-24 months for a mean of 13 years. We considered subjects who developed AD and non-converters. Results: Clock T3111C was detected in 47\% of cases, Per2 $\mathrm{C} 111 \mathrm{G}$ in $19 \%$ of cases. ApoE \&4 carriers presented higher risk of heart disease; Clock C-carriers were more frequently smokers than non C-carriers. During the follow-up, 17 patients progressed to AD. Age at baseline, $A p o E \varepsilon 4$ and dyslipidemia increased the risk of conversion to AD. ApoE $\varepsilon 4$ carriers with history of dyslipidemia showed higher risk to convert to AD compared to ApoE $\varepsilon 4-$ groups and $A p o E \varepsilon 4+$ without dyslipidemia patients. Clock C-carriers with history of blood hypertension had a higher risk of conversion to AD. Conclusions: ApoE and Clock T3111C seem to interact with cardiovascular risk factors in SCD and MCI patients influencing the progression to AD.
\end{abstract}

Keywords: Alzheimer's disease; subjective cognitive decline; mild cognitive impairment; clock genes; Clock; ApoE; cardiovascular risk factors

\section{Introduction}

Alzheimer's disease (AD) is characterized by a slow but progressive trend, with a presymptomatic phase that can last from years to decades [1]. Subjective Cognitive Decline (SCD) is defined as a self-experienced persistent decline in cognitive capacity in comparison with the subject's previously status, during which the subject has normal performance on standardized cognitive tests [2]. Mild cognitive impairment (MCI) concerns an objective cognitive impairment with minimal impact on instrumental activity of daily living [3], and it is considered an intermediate phase between normal cognition and dementia. MCI is associated with an increased risk of positive AD biomarkers and with 
an annual conversion rate of $5 \%-17 \%$ to $\mathrm{AD}[4,5]$. For $\mathrm{SCD}$, the annual conversion rate (ACR) to MCI is $3.6 \%-6.6 \%$, while it is $1.5 \%-2.3 \%$ to dementia $[4,6]$.

A growing amount of evidence has underlined the importance of cardiovascular health on the risk of developing AD [7-9]. It has been reported that approximately one-third of AD cases worldwide may be attributable to cardiovascular risk factors, including hypertension, obesity, diabetes, smoking, and physical inactivity [10].

In addition, it is well known that genetic aspects play a central role in development of AD [11]. Apolipoprotein E e4 carrier status ( $A p o E$ e4) is a well-defined genetic risk factor, and recently, current research has been focused on clock genes. Clock (Circadian Locomotor Output Cycle Kaput, chromosome 4q12) and Per2 (Period2, chromosome 2q37.3) are part of the transcriptional-translational feedback loops regulating circadian rhythm [12]. Several polymorphisms of these genes have been recently studied to elucidate their role in sleep-wake cycle alterations, aging, psychiatric disturbance and neurodegeneration $[13,14]$. Moreover, Clock and Per2 polymorphisms have been associated with overweight and glucose and lipid metabolism impairments $[15,16]$. Some studies have investigated the possible role of the Clock T3111C polymorphism on the quality of aging in very elderly patients [17], while other studies focused on influence of Per2 C111G polymorphism on lipid metabolism in adults with metabolic syndrome [16]. On the basis of the above-mentioned initial findings about of the influence of these polymorphisms on cardiovascular profile, the aim of the present study was to define the interaction between Clock T3111C and Per2 C111G, ApoE \&4, and cognitive function, in relation to cardiovascular risk factors, in SCD and $\mathrm{MCI}$ patients and in the progression to AD.

\section{Materials and Methods}

\subsection{Participants and Clinical Assessment}

As part of a longitudinal, clinical-neuropsychological-genetic survey on SCD and MCI, we included 74 consecutive spontaneous patients who self-referred to the Centre for Alzheimer's Disease and Adult Cognitive Disorders of Careggi Hospital in Florence between April 1996 and May 2014. All participants underwent a comprehensive family and clinical history, general and neurological examination, extensive neuropsychological investigation, estimation of premorbid intelligence, as well as assessment of depression. A positive family history was defined as one or more first-degree relatives with documented cognitive decline. Sleep quality was assessed according to anamnestic data: we considered as "poor sleepers" patients who had difficulties in falling asleep or woke up early or have frequent sleep interruptions; patients that did not report sleep disturbances were classified as "good sleepers". For this study, inclusion criteria were: (1) complaining of cognitive decline with a duration of $\geq 6$ months; (2) normal functioning on the Activities of Daily Living and the Instrumental Activities of Daily Living scales; (3) unsatisfied criteria for dementia at baseline [18]; (4) attainment of the clinical endpoint, i.e., conversion to AD according to the NIA-AA [18] criteria during follow up, regardless of follow-up duration; (5) a follow-up time of more than 2 years from the baseline visit for those patients who did not develop AD. Exclusion criteria were: (1) history of head injury, current neurological and/or systemic disease, symptoms of psychosis, major depression, alcoholism or other substance abuse; (2) the complete data loss of patients' follow-up; (3) progression to dementia other than AD.

From the initial sample, we excluded six patients: two patients had a follow-up shorter than 2 years; two diagnosed with psychiatric disturbance, and one with Fronto-Temporal Dementia, according to Neary criteria [19]; one patient received a diagnosis of Vascular Dementia [20]. Therefore, in the end 68 patients were included.

We divided this sample into two groups: 41 patients classified as SCD, according to the terminology proposed by the Subjective Cognitive Decline Initiative (SCD-I) Working Group [2] (i.e., presence of a self-experienced persistent decline in cognitive capacities with normal performance on standardized cognitive tests); 27 patients classified as MCI according to (NIA-AA) criteria for the diagnosis of MCI [3] 
(i.e., evidence of lower performance in one or more cognitive domains with preserved independence of function in daily life).

All patients underwent clinical and neuropsychological follow-up every 12 or 24 months. All of them were genotyped for ApoE (Apolipoprotein E), Clock and Per2.

On the basis of progression to AD during the follow-up, patients were classified respectively into converters and non-converters. All subjects gave their informed consent for inclusion before they participated in the study. The study was conducted in accordance with the Declaration of Helsinki, and the protocol was approved by the Ethics Committee (DSM study).

\subsection{Neuropsychological Assessment}

All patients were evaluated by means of an extensive neuropsychological battery [21]. The battery consisted of global measurements [Mini-Mental State Examination (MMSE)], tasks exploring verbal and spatial short- term memory (Digit Span; Corsi Tapping Test) and verbal long-term memory [Five Words and Paired Words Acquisition (FWA, PWA); recall after 10min (FWR10, PWR10); recall after 24-h (FWR24, PWR24); Babcock Short Story Immediate and Delayed Recall (BS, BSR)]; language (Token Test; Category Fluency Task); and visuo-motor functions (Copying Drawings) [21]. Visuospatial abilities were also evaluated by Rey-Osterrieth Complex Figure copy, and visuospatial long-term memory was assessed by means of recall of Rey-Osterrieth Complex Figure test [22]; attention/executive function was explored by means of Dual Task [23], Phonemic Fluency Test [24], and Trail Making Test [25]. Everyday memory was assessed by means of Rivermead Behavioral Memory Test (RBMT) [26]. All raw test scores were adjusted for age, education and gender according to the correction factor reported in validation studies for the Italian population [21-26]. In order to estimate pre-morbid intelligence, all patients were given the TIB ("Test di Intelligenza Breve") [27], an Italian version of the National Adult Reading Test [28]. The presence and severity of depressive symptoms was evaluated by means of the 22-item Hamilton Depression Rating Scale (HRSD) [29].

\subsection{Apolipoprotein E ع4, Clock T3111C and Per2 C111G Genotyping}

A standard automated method (QIAcube, QIAGEN) was used to isolate DNA from peripheral blood samples. ApoE genotypes were investigated by high resolution melting analysis (HRMA). Two sets of PCR primers were designed to amplify the regions encompassing rs7412 [NC_000019.9:g.45412079C>T] and rs429358 (NC_000019.9:g.45411941T>C). The samples with known ApoE genotypes, which had been validated by DNA sequencing, were used as standard references. The ApoE genotype was coded as ApoE $\varepsilon 4-$ (no ApoE $\varepsilon 4$ alleles) and ApoE $\varepsilon 4+$ (presence of one or two ApoE $\varepsilon 4$ alleles).

The analyses of Clock and Per2 were performed using HRMA in order to detect the 3111T/C Clock polymorphism using primers as reported [30] and the Per2 C111G polymorphism with the following primers Forward 5'-ACAGAAAGAGTCAAATGGGTGC- $3^{\prime}$, Reverse $5^{\prime}$-TGTCCACATCTTCCTGCAGT-3' with Annealing temperature $60^{\circ} \mathrm{C}$.

\subsection{Statistical Analysis}

Patient groups were characterized using means and standard deviations (SD). We tested for the normality distribution of the data using the Kolmogorov-Smirnov test. Depending on the distribution of our data, we used t-test or non-parametric Mann-Whitney U Tests for between-groups' comparisons. We used chi-square test to compare categorical data. We analyzed survival curves using the Kaplan-Meier estimator. Finally, we used logistic regression to analyze the role of some cardiovascular risk factors in worsening cognition. All statistical analyses were performed with SPSS software v.25 (SPSS Inc., Chicago, IL, USA). The significance level was set at $p<0.05$. 


\section{Results}

\subsection{Participants and Clinical Assessment}

In the whole cohort, 32 of 68 patients (47\%) were Clock C carriers (29 TC, 3 CC), while 13 of 68 (19\%) were Per2 G carriers (13 CG, 0 GG); 7 of 68 (10\%) carried both Clock C and Per2 G alleles. The genotypic distributions of the Clock and Per2 genes in this sample were in Hardy-Weinberg equilibrium (Clock T3111C $\chi^{2}=0.91, \mathrm{p}>0.05$; Per 2 C111G $\left.\chi^{2}=0.77, p>0.05\right)$. The prevalence of Clock T3111C and Per2 C111G polymorphisms did not significantly differ between SCD and MCI (Clock T3111C: 51.2\% in SCD and 40.7\% in MCI; Per2 C111G: $19.5 \%$ in SCD and 18.5\% in MCI); moreover, there were not any differences in the prevalence of both Clock C and Per2 G carriers in SCD and MCI groups. (Table 1).

Table 1. Comparison between prevalence of Clock and Per2 polymorphisms in SCD and MCI individuals.

\begin{tabular}{cccc}
\hline Features & SCD & MCI & $p$ \\
\hline Per2 G carriers-units (\%) & $8(19.5 \%)$ & $5(18.5 \%)$ & 0.919 \\
Clock C carriers-units (\%) & $21(51.2 \%)$ & $11(40.7 \%)$ & 0.397 \\
Per2 G carriers and & $4(9.75 \%)$ & $3(8.10 \%)$ & 0.843 \\
Clock C carriers-units (\%) & & &
\end{tabular}

$p$ indicates level of significance for comparison between groups (statistical significance at $p<0.05$, in bold characters).

There were no differences between Clock $\mathrm{C}$ carriers and non $\mathrm{C}$ carriers with regards to age at onset of symptoms, age at baseline visit, disease duration, sex, family history of AD, years of education, TIB, MMSE, and ApoE $\varepsilon 4$ allele status. With respect to $C V$ risk factors, there was a higher proportion of smokers in Clock C carriers than in non C carriers $\left(19.40 \%\right.$ vs. $\left.2.80 \%, \chi^{2}=4,892, p=0.027\right)$ (Table 2).

Comparing Per2 G carriers and non G carriers, there were no differences in age at onset of symptoms, age at baseline visit, disease duration, sex, family, MMSE, and ApoE $\varepsilon 4$ allele status. Per2 G carriers had lower premorbid intelligence score on TIB $(104.29 \pm 10.74$ vs. $109.98 \pm 8.06, p=0.049)$, less years of education $(7 \pm 3.05$ vs $10.73 \pm 4.51, p=0.007)$, and lower frequency of family history of AD $\left(15.38 \%\right.$ vs. $\left.60 \%, \chi^{2}=8.37, p=0.004\right)$ (Table 2$)$. There were no differences in CV risk factors proportion between $\mathrm{G}$ and non $\mathrm{G}$ carriers.

We did not find any differences in sleep quality between Clock $\mathrm{C}$ carriers and Clock non $\mathrm{C}$ carriers $\left(\chi^{2}=0.136, p=0.454\right)$, neither between Per $2 \mathrm{G}$ carriers and Per 2 non G carriers $\left(\chi^{2}=0.879, p=0.273\right)$ (Table 2).

$A p o E \varepsilon 4+$ patients have a higher proportion of history of heart disease than $A p o E \varepsilon 4-(8.70 \%$ vs. $\left.0.00 \%, \chi^{2}=3.944, p=0.047\right)$. We did not find any statistically significant difference between ApoE $\varepsilon 4+$ and ApoE $\varepsilon 4-$ as far age at onset of symptoms, age at baseline evaluation, disease duration (time from onset of symptoms and baseline evaluation), follow-up time, familiarity, sex, education, and MMSE (Table 2). 
Table 2. Demographic data according to Per2, Clock, ApoE4.

\begin{tabular}{|c|c|c|c|c|c|c|c|c|c|}
\hline \multirow{2}{*}{ Features } & \multicolumn{3}{|c|}{ Per2 } & \multicolumn{3}{|c|}{ Clock } & \multicolumn{3}{|c|}{ ApoE } \\
\hline & non $G(n=55)$ & $G(n=13)$ & $p$ & non $C(n=36)$ & $C(n=32)$ & $p$ & $\varepsilon 4-(n=45)$ & $\varepsilon 4+(n=23)$ & $p$ \\
\hline SCD (\% within SCD) & $80.5 \%$ & $19.5 \%$ & - & $48.8 \%$ & $51.2 \%$ & - & $68.3 \%$ & $31.7 \%$ & - \\
\hline MCI (\% within MCI) & $81.5 \%$ & $18.5 \%$ & - & $59.3 \%$ & $40.7 \%$ & - & $63 \%$ & $37 \%$ & - \\
\hline $\begin{array}{l}\text { Conversion to AD (SCD) (\% in } \\
\text { consideration of carrier status) }\end{array}$ & $0.0 \%$ & $25.0 \%$ & - & $5.0 \%$ & $4.8 \%$ & - & $3.6 \%$ & $7.7 \%$ & - \\
\hline $\begin{array}{l}\text { Conversion to AD (MCI) (\% in } \\
\text { consideration of carrier status) }\end{array}$ & $59.1 \%$ & $40.0 \%$ & - & $62.5 \%$ & $45.5 \%$ & - & $35.3 \%$ & $90 \%$ & - \\
\hline Age at baseline $( \pm S D)$ in years & $64.03 \pm 9.12$ & $65.22 \pm 7.26$ & 0.739 & $64.29 \pm 9.33$ & $63.69 \pm 8.60$ & 0.777 & $63.38 \pm 9.08$ & $65.38 \pm 8.75$ & 0.354 \\
\hline Age at onset $( \pm S D)$ in years & $59.69 \pm 10.02$ & $62.38 \pm 7.96$ & 0.562 & $60.56 \pm 10.87$ & $59.25 \pm 8.64$ & 0.694 & $59.38 \pm 10.12$ & $61.83 \pm 9.17$ & 0.228 \\
\hline Follow-up time $( \pm S D)$ in years & $10.87 \pm 4.23$ & $12.60 \pm 4.68$ & 0.234 & $11.34 \pm 4.31$ & $10.90 \pm 4.45$ & 0.815 & $13.00 \pm 4.63$ & $10.54 \pm 4.71$ & 0.065 \\
\hline Disease duration $( \pm S D)$ in years & $4.36 \pm 3.63$ & $2.83 \pm 2.33$ & 0.090 & $3.73 \pm 3.65$ & $4.44 \pm 3.18$ & 0.195 & $4.00 \pm 4.85$ & $3.55 \pm 2.00$ & 0.800 \\
\hline Sex (females, males) & 37. 17 & 10.3 & 0.552 & 26. 10 & 22. 10 & 0.754 & 33,12 & 15,8 & 0.487 \\
\hline Family history of AD (\%) & $61.11 \%$ & $15.38 \%$ & $0.003 *$ & $41.66 \%$ & $62.5 \%$ & 0.086 & $43.18 \%$ & $65.22 \%$ & 0.105 \\
\hline Education in years $( \pm \mathrm{SD})$ & $10.72 \pm 4.55$ & $7 \pm 3.05$ & 0.007 * & $9.86 \pm 4.80$ & $10.19 \pm 4.21$ & 0.737 & $9.73 \pm 4.51$ & $10.57 \pm 4.54$ & 0.455 \\
\hline MMSE $( \pm \mathrm{SD})$ & $28.46 \pm 1.66$ & $28.31 \pm 1.49$ & 0.583 & $28.09 \pm 1.78$ & $28.81 \pm 1.33$ & 0.064 & $28.34 \pm 1.78$ & $28.595 \pm 1.26$ & 0.839 \\
\hline ApoE ع4 (\%) & $35.18 \%$ & $23.07 \%$ & 0.404 & $30.55 \%$ & $37.5 \%$ & 0.546 & - & - & - \\
\hline Diabetes (\%) * & $5.60 \%$ & $7.70 \%$ & 0.770 & $8.30 \%$ & $3.10 \%$ & 0.362 & $2.20 \%$ & $13.00 \%$ & 0.730 \\
\hline Hypertension (\%) * & $22.20 \%$ & $23.10 \%$ & 0.947 & $25.00 \%$ & $18.8 \%$ & 0.535 & $22.20 \%$ & $21.70 \%$ & 0.964 \\
\hline Dyslipidemia (\%) * & $30.80 \% \%$ & $23.10 \%$ & 0.585 & $30.60 \%$ & $26.70 \%$ & 0.728 & $34.10 \%$ & $18.20 \%$ & 0.178 \\
\hline Heart Disease (\%) * & $3.80 \%$ & $0.00 \%$ & 0.477 & $2.90 \%$ & $3.10 \%$ & 0.949 & $0.00 \%$ & $8.70 \%$ & 0.047 \\
\hline Smoking (\%) * & $11.30 \%$ & $7.70 \%$ & 0.703 & $2.80 \%$ & $19.40 \%$ & 0.027 & $6.80 \%$ & $17.40 \%$ & 0.179 \\
\hline
\end{tabular}

Values quoted in the table are mean ( \pm SD) or (\%) or units. $p$ indicates level of significance for comparison between groups (statistical significance at $p<0.05$, in bold characters). ${ }^{*}$ every risk factor was evaluated at the baseline for statistical purpose. 


\subsection{Description of Sample at Follow-Up}

During the follow-up, 17 patients (25\%, 2 SCD and $15 \mathrm{MCI}$ ) converted to AD (converters) while 51 patients did not progress to $\mathrm{AD}$ (non-converters). Mean conversion time was $4.73 \pm 3.91$ years (range: $1.41-14.01, \mathrm{IQR}=3.71$ years). Mean follow-up time of non-converters was $13.03 \pm 4.48$ years (range: 4.06-23.74, IQR $=6.73$ years). There were no differences between converters and non-converters with respect to disease duration, sex, family history of $\mathrm{AD}$, years of education, and MMSE at baseline. Converters had higher age at the onset of symptoms ( $68.20 \pm 7.49$ vs. $61.25 \pm 6.21, p=0.016)$, age at baseline $(71.60 \pm 6.19$ vs. $63.42 \pm 6.96, p=0.003)$ and greater proportion of ApoE $\varepsilon 4(56.25 \%$ vs. $25.49 \%$, $\chi^{2}=5.22, p=0.022$ ). (Table 3).

Table 3. Comparison of demographic and clinical data between converters and non-converters.

\begin{tabular}{|c|c|c|c|}
\hline Features & Converters $(n=17)$ & Non Converters $(n=51)$ & $p$ \\
\hline Prevalence Per2 G (\%) & $23.5 \%$ & $17.6 \%$ & 0.593 \\
\hline Prevalence Clock C (\%) & $35.3 \%$ & $51.0 \%$ & 0.262 \\
\hline Age at baseline $( \pm S D)$ in years & $70.7( \pm 6.3)$ & $61.8( \pm 8.6)$ & $<0.01$ \\
\hline Age at onset $*( \pm S D)$ in years & $67.4( \pm 7.5)$ & $57.9( \pm 9.4)$ & $<0.01$ \\
\hline Follow-up time $( \pm S D)$ in years & $9.6( \pm 4.7)$ & $13.03( \pm 4.5)$ & $<0.01$ \\
\hline Disease duration $( \pm S D)$ in years & $3.3( \pm 3.6)$ & $4.0( \pm 4.3)$ & 0.602 \\
\hline Sex (females, males) & 12,5 & 36,15 & 1.000 \\
\hline Family history of AD (\%) & $52.9 \%$ & $51.0 \%$ & 0.889 \\
\hline Education in years $( \pm S D)$ & $8.4( \pm 3.9)$ & $10.6( \pm 4.6)$ & 0.059 \\
\hline MMSE $( \pm S D)$ & $28.1( \pm 1.1)$ & $28.5( \pm 1.8)$ & 0.399 \\
\hline ApoE \&4 (\%) & $58.8 \%$ & $25.5 \%$ & 0.012 \\
\hline Hypertension (\%) & $29.4 \%$ & $19.6 \%$ & 0.399 \\
\hline Diabetes (\%) & $11.8 \%$ & $3.9 \%$ & 0.234 \\
\hline Dyslipidemia (\%) & $47.1 \%$ & $22.4 \%$ & 0.053 \\
\hline Heart disease $(\%)$ & $11.8 \%$ & $0.0 \%$ & 0.014 \\
\hline Smoking (current) (\%) & $0.0 \%$ & $14.0 \%$ & 0.103 \\
\hline Chronic kidney disease (\%) & $0.0 \%$ & $0.0 \%$ & - \\
\hline
\end{tabular}

Values quoted in the table are mean $( \pm \mathrm{SD}$ ) or $(\%) . p$ indicates level of significance for comparison between groups (statistical significance at $p<0.05$, in bold characters). ${ }^{*}$ onset of memory problems (not overt dementia).

There were no significant differences between converters and non-converters in the prevalence of Clock T3111C (35.3\% vs. $\left.51.0 \%, \chi^{2}=1.26, p=0.262\right)$ and Per2 C111G $\left(23.5 \%\right.$ vs. $17.6 \%, \chi^{2}=0.285$ $p=0.593$ ) polymorphism (Table 3 ).

In order to ascertain the effects of cardiovascular risk factors on the conversion to $\mathrm{AD}$, we performed a proportional hazards regression analysis considering conversion time as time and "conversion to $\mathrm{AD}^{\prime \prime}$ as dependent variable. We considered as covariates age at onset, age at baseline, ApoE, Clock and Per2 genotype, hypertension, diabetes, dyslipidemia, heart disease, and smoking habit. Dyslipidemia $(p=0.041, \mathrm{HR}=3.08,95 \%$ I.C. $=1.05: 9.09)$, age at baseline $(p=0.001, \mathrm{HR}=1.16,95 \%$ I.C. $=1.07: 1.27)$ and ApoE $\varepsilon 4(p=0.001, \mathrm{HR}=6.21,95 \%$ I.C. $=2.04: 18.9)$ were statistically significantly associated with an increased likelihood of conversion to $\mathrm{AD}$ (Table 4). 
Table 4. Proportional hazards regression analysis.

\begin{tabular}{cccccc}
\hline & B & $p$ & HR & \multicolumn{2}{c}{ 95\% C.I. } \\
\hline \multicolumn{2}{c}{ Whole sample } & & & Lower & Upper \\
\hline ApoE e4 & 1.826 & 0.001 & 6.212 & 2.045 & 18.872 \\
\hline Age at baseline & 0.151 & 0.001 & 1.163 & 1.067 & 1.269 \\
\hline Dyslipidemia & 0.126 & 0.041 & 3.083 & 1.045 & 9.099 \\
\hline Clock C carriers & & & & & \\
\hline Hypertension & 3.265 & 0.025 & 26.18 & 1.510 & 454.039 \\
\hline Clock non C carriers & & & & & \\
\hline Age at baseline & 0.204 & 0.002 & 1.23 & 1.078 & 1.394 \\
\hline ApoE & 2.111 & 0.009 & 8.25 & 1.712 & 39.791 \\
\hline
\end{tabular}

Regression Coefficients (B), $p$-value ( $p$ ), Hazard Ratio (OR) and 95\% Confidence Intervals (95\% CI) for covariates included in the proportional hazards regression model are reported (significant differences at $p<0.05$ ).

\subsection{Relationship between ApoE and Dyslipidemia}

In order to explore the relationship between dyslipidemia and ApoE genotype, we divided the sample according to ApoE $\varepsilon 4$ status ( $\varepsilon 4+$ and $\varepsilon 4-)$. In the $\varepsilon 4+$ sample, a Kaplan-Meier survival analysis showed significant difference in survival distributions between patients with history of dyslipidemia and patients without history of dyslipidemia $\left(x^{2}=4.42, p=0.036\right)$, as $100 \%$ of dyslipidemic patients and $29.4 \%$ of non-dyslipidemic patients converted to AD (Figure 1a). When we performed the same analysis on the $\varepsilon 4$ - sample, we found no statistically significant effect of dyslipidemia on rate of progression to $\operatorname{AD}\left(\chi^{2}=1.92, p=0.166\right)$ (Figure $\left.1 b\right)$.

Finally, we ranked the whole sample according to history of dyslipidemia and ApoE genotype (non-dyslipidemic/ $\varepsilon 4-, \mathrm{n}=29$; non-dyslipidemic/ $\varepsilon 4+, \mathrm{n}=17$; dyslipidemic/ $\varepsilon 4-, \mathrm{n}=15$; dyslipidemic/ $\varepsilon 4$, $\mathrm{n}=4$ ) and Kaplan-Meier survival analysis was conducted to compare the proportions of conversions in the four different groups. Patients in dyslipidemic/ $/ \varepsilon 4+$ group had a higher rate of conversion to AD compared to non-dyslipidemic/ $\varepsilon 4-\left(\chi^{2}=25.47, p<0.001\right)$, non-dyslipidemic/ $/ \varepsilon 4+\left(\chi^{2}=4.42, p=0.036\right)$ and dyslipidemic/ $\varepsilon 4-\left(\chi^{2}=7.64, p=0.006\right)$. Proportion of conversion in non-dyslipidemic/ $\varepsilon 4+$ was higher as compared to non-dyslipidemic/ $\varepsilon 4-\left(\chi^{2}=3.73, p=0.05\right)$. There was no significant difference

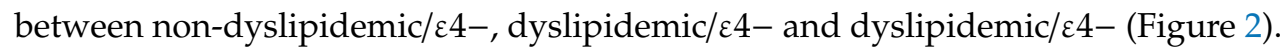

\subsection{Relationship between Clock and Per2 and Risk Factors on Progression to $A D$}

In order to explore the relationship between $\mathrm{CV}$ risk factors and Clock polymorphism, we divided the sample according to Clock genotype status ( $C$ carriers and non $C$ carriers). For each sample, we performed a proportional hazards regression analysis considering conversion time as time; "conversion to $\mathrm{AD}^{\prime \prime}$ as dependent variable; and age at onset, age at baseline, ApoE genotype, hypertension, diabetes, dyslipidemia, heart disease, and smoking habit as covariates. In the $C$ carriers sample, hypertension at baseline ( $p=0.025, \mathrm{HR}=3.625)$ was statistically significantly associated with an increased likelihood of conversion to $\mathrm{AD}$ (Table 4). In the Clock non C carriers, none of the CV factors were included in the model as only age at baseline was statistically significantly associated with high risk of progression to $\mathrm{AD}$. 

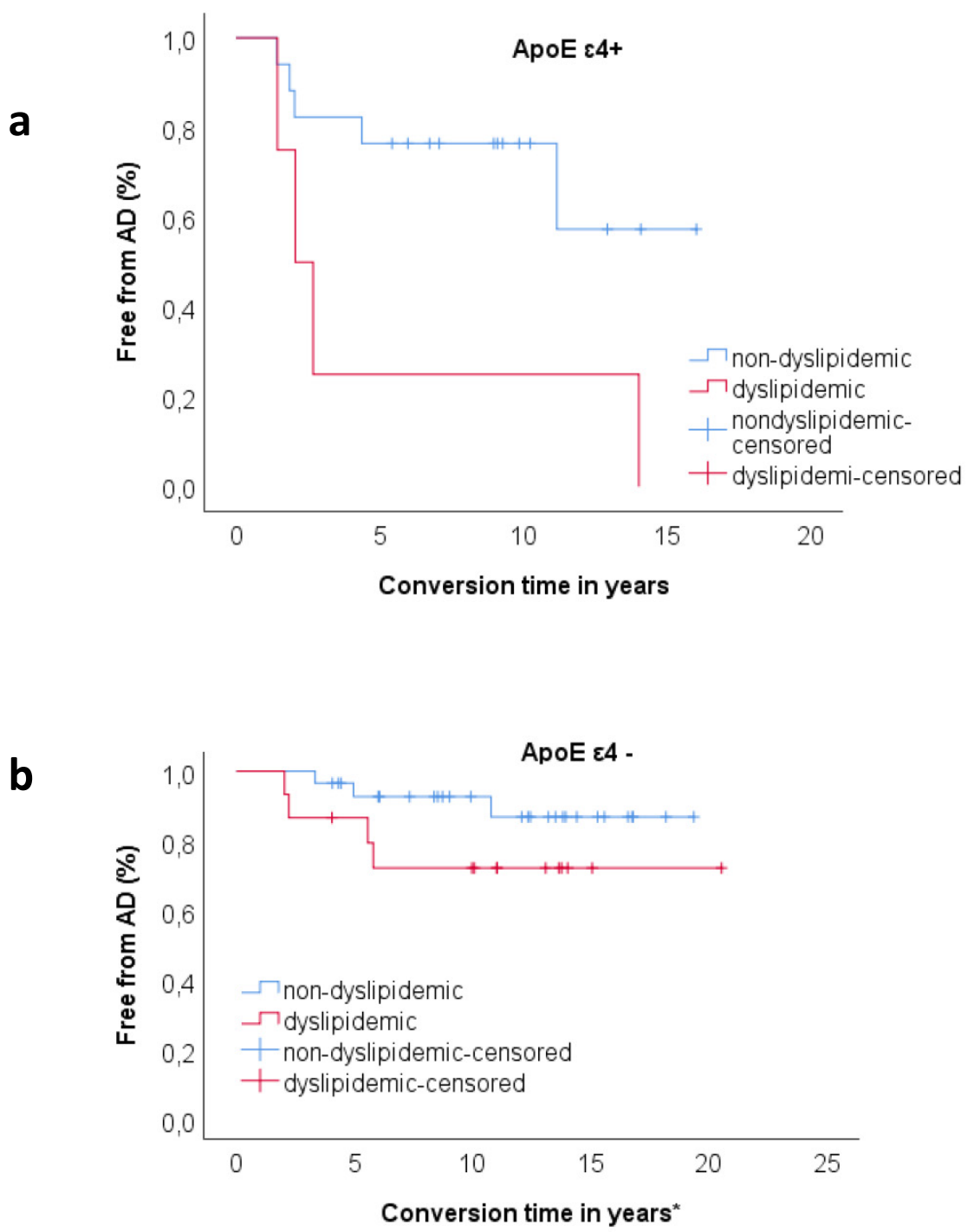

Figure 1. (a) Kaplan-Meier survival analysis for comparisons of proportion of progression to AD between dyslipidemic $(\mathrm{n}=4)$ and non-dyslipidemic $(\mathrm{n}=17)$ patients in ApoE $\varepsilon 4+$ carrier group. Proportion of progression was higher in dyslipidemic group $(100.00 \%)$ compared to non-dyslipidemic $(29.40 \%)$. The pairwise log rank comparisons showed significant difference in survival distributions for the dyslipidemic vs. non-dyslipidemic $\left(\chi^{2}=4.42, p=0.036\right)$. (b) Kaplan-Meier survival analysis for comparisons of proportion of progression to AD between dyslipidemic $(n=15)$ and non-dyslipidemic ( $\mathrm{n}=29$ ) patients in ApoE $\varepsilon 4$ - carrier group. The pairwise log rank comparisons showed no significant difference in survival distributions for the dyslipidemic vs. non-dyslipidemic $\left(\chi^{2}=1.92, p=0.166\right)$ * For censored cases (non-converters), conversion time indicates follow-up time. 


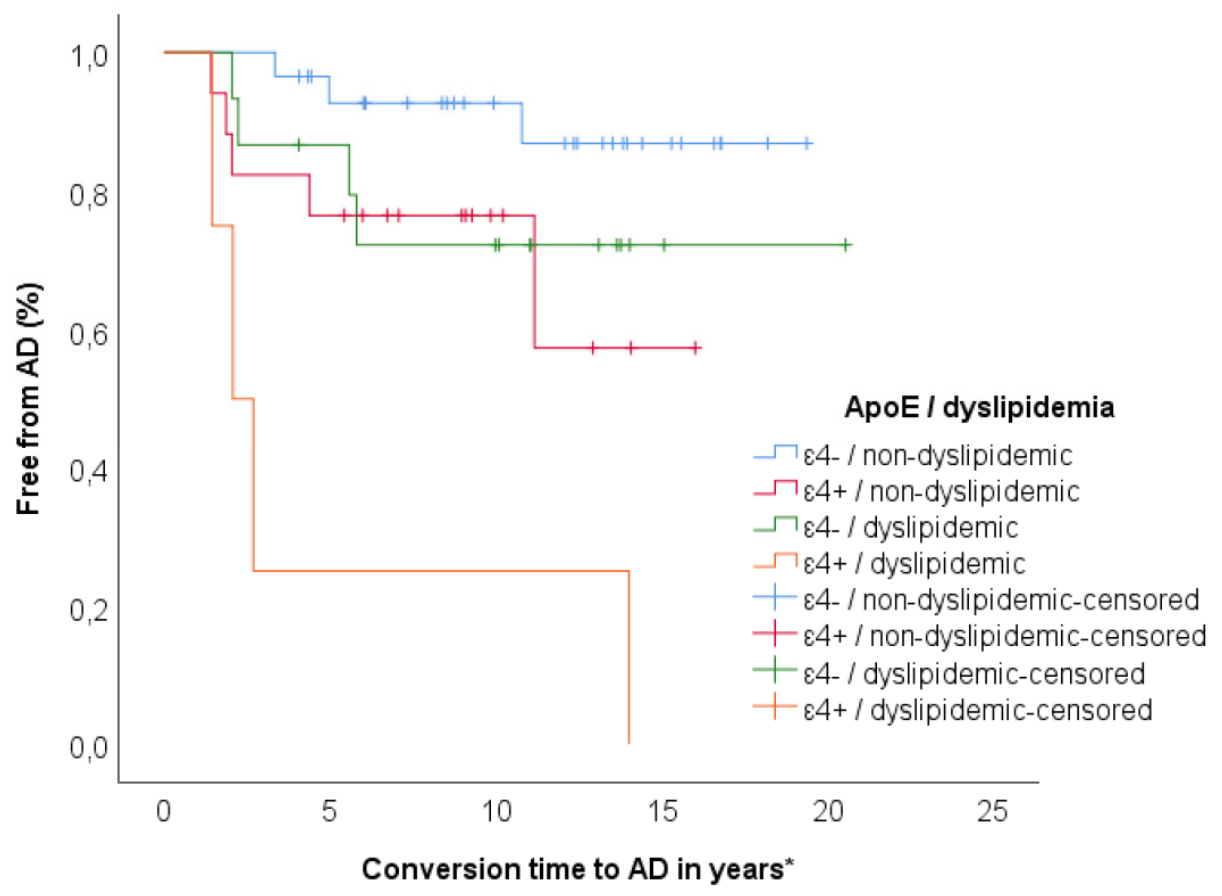

Figure 2. Kaplan-Meier survival analysis for comparisons of patients ranked according to history

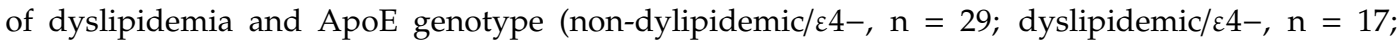
non-dylipidemic/high/ $\varepsilon 4+, \mathrm{n}=15$; dylipidemic/ $\varepsilon 4+, \mathrm{n}=4)$. Proportion of progression was higher in dylipidemic/ $\varepsilon 4+(100.00 \%)$ compared to non-dylipidemic/ $\varepsilon 4-\left(10.30 \%, \chi^{2}=25.47, p<0.001\right)$, non-dylipidemic/ $\varepsilon 4+\left(29.40 \%, \chi^{2}=4.42, p=0.036\right)$, and dylipidemic $/ \varepsilon 4-\left(26.7 \%, \chi^{2}=7.64, p=0.006\right)$.

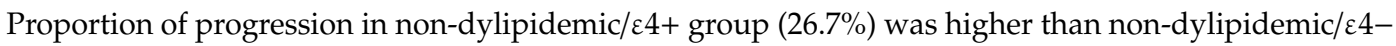
$\left(10.3 \%, \chi^{2}=3.73, p=0.05\right)$. * For censored cases (non converters) conversion time indicates follow-up time.

A Kaplan-Meier survival analysis showed significant difference in survival distributions between patients with history of hypertension and patients without history of hypertension $\left(\chi^{2}=4.42, p=\right.$ 0.036 ) only in the Clock $C$ carriers sample (Figure $3 a$ ). In this group, $50 \%$ of hypertensive patients and $11.5 \%$ of non-hypertensive patients converted to $\mathrm{AD}$. When we performed the same analysis on the Clock non $C$ carriers sample, we found no statistically significant effect of hypertension on rate of progression to AD; (Figure 3b).

We performed the proportional hazard regression analysis ranking patients according to Per2 polymorphism, including as covariates age at onset, age at baseline, ApoE genotype, hypertension, diabetes, dyslipidemia, heart disease, and smoking. In the G carriers sample, none of the covariates showed a statistically significant effect on risk of conversion to AD. In the Per2 non G carriers, none of the CV factors were included in the model as only age at baseline was statistically significantly associated with high risk of progression to $\mathrm{AD}(p=0.010, \mathrm{HR}=1.185)$. 
a

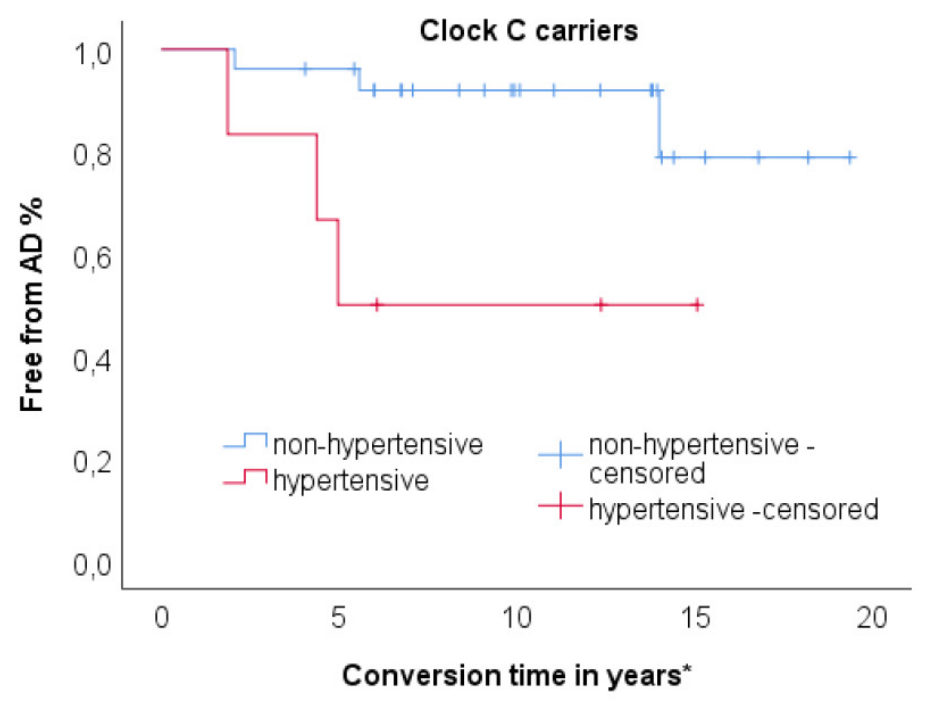

b

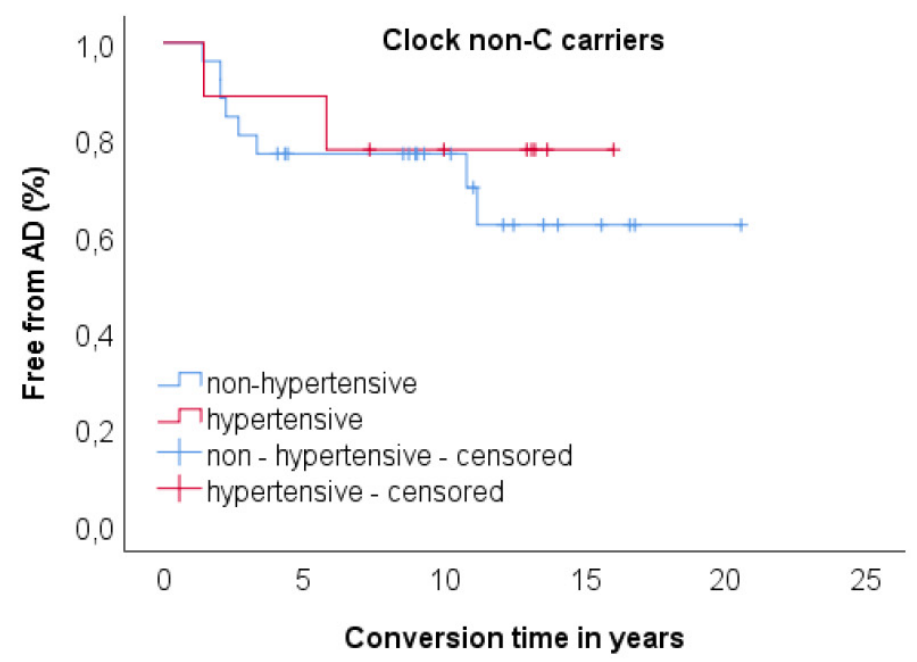

Figure 3. (a) Kaplan-Meier survival analysis for comparisons of proportion of progression to AD between hypertensive $(n=6)$ and non-hypertensive $(n=26)$ patients in Clock $C$ carriers group. Proportion of progression was higher in hypertensive group $(50.00 \%)$ compared to non-hypertensive (11.50\%). The pairwise log rank comparisons showed significant difference in survival distributions for the hypertensive vs. non-hypertensive $\left(\chi^{2}=5.77, p=0.017\right)$. (b) Kaplan-Meier survival analysis for comparisons of proportion of progression to AD between hypertensive $(n=9)$ and non-hypertensive $(n$ =26) patients in Clock non C carriers group. The pairwise log rank comparisons showed no significant difference in survival distributions for the hypertensive vs. non-hypertensive $\left(\chi^{2}=0.323, p=0.570\right)$ * For censored cases (non converters) conversion time indicates follow-up time.

\section{Discussion}

This study investigated the interaction between genetic features (Clock T3111C, Per2 C111G polymorphisms, and ApoE genotype) and cardiovascular risk factors in a sample of SCD and MCI patients. We are not aware of any previous studies exploring this topic on these groups of patients.

The frequency of these polymorphisms in our cohort was similar to prevalence data reported in a previous study on healthy Italian population [31]. 
We found that Clock T3111C carriers were more frequent smokers compared to non-carriers of the polymorphism. Our result may suggest an implication also of Clock T3111C on nicotine dependence. Other researches have shown that clock genes are associated with substance abuse, including alcohol, cocaine and cannabis [32]. In fact, circadian genes have a direct role in in the regulation of dopaminergic transmission, especially in reward circuitry. This evidence could represent the biological substrate for the role of Clock genes in the development of addicted behaviours [33,34].

We found a correlation between Per 2 C111G polymorphism with years of education and family history of AD. This result confirms previous evidence by our group [35], but it is difficult to interpret this finding due to the absence of previous works on this issue.

For future researches on wider sample, we will aim to clarify our current findings. With regard to cardiovascular risk factors, we did not find any association with Per2 C111G.

We found that $A p o E \varepsilon 4$ carriers had more frequent history of heart disease than $\varepsilon 4$ non carriers. Our result is supported by previous studies. In particular, two different meta-analysis $[36,37]$ showed a different distribution of coronary disease risk according to ApoE genotype. Furthermore, this association could be independent from other cardiovascular risk factors, as we did not find any correlation with diabetes, dyslipidemia, smoking, or hypertension.

A multivariate analysis showed that age at baseline, $A p o E$ e4 and dyslipidemia increase the risk of progression to $\mathrm{AD}$.

With regard to age, this is not surprising as it is recognized to be the major risk factor for $\mathrm{AD}[38,39]$.

The effect of $A p o E \varepsilon 4$ allele on risk of progression to AD has been widely demonstrated by previous studies [40-43].

Last, lipid disorders have been said to have a role in cognitive impairment and their treatment has been studied as a prevention tool, but evidence about this topic is not yet conclusive [44].

The interaction between $A p o E \& 4$ and dyslipidemia on cognition is not yet completely understood [36]. In order to explore this point, we ranked the patients according to ApoE genotype. The effect of dyslipidemia on progression to $\mathrm{AD}$ was confirmed only in $A p o E \varepsilon 4$ carriers. Furthermore, we showed that patients who were $A p o E \varepsilon 4$ carriers and had history of dyslipidemia showed higher risk to convert to AD both compared to ApoE\&4- groups and $A p o E \varepsilon 4+$ without dyslipidemia patients. According to this analysis, dyslipidemia could be synergistic with E4 carrier status in contributing to AD pathogenesis as reported by other authors [44].

Previous studies suggested an association between Clock gene polymorphisms with different cardiovascular risk factors, as well as with the cognitive state [15-17]. In order to investigate a possible interaction between Clock T3111C and cardiovascular risk factors, we ranked patients according to Clock genotype. We found that Clock $\mathrm{C}$ carriers with history of blood hypertension had a higher risk of conversion to $\mathrm{AD}$ than Clock $\mathrm{C}$ carriers without hypertension. This difference was not detected in the Clock non C carrier group. No other work, to the best of our knowledge, has previously studied the possible influence of Clock gene polymorphisms on the effect of hypertension in conversion to AD in this particular group of patients.

A limitation of this study is the small size of our cohort. In future we aim to expand our sample, also including a healthy control group, to support our present results.

Secondly, the lack of quantitative data about dyslipidemia and hypertension did not allow us to understand if our results might be different according to the level of blood lipids and blood pressure. Another limitation of this study is that AD diagnosis was not supported by AD biomarkers. Future researches including cerebrospinal fluid amyloid beta, tau and p-tau levels or neuroimaging data, as amyloid PET, could provide interesting and additional information. Finally, as it is a single-center study, there may be biases with regard to assessment and diagnosis procedures. On the other hand, this study has some remarkable strengths. First of all, to the best of our knowledge, this is the first prospective study that assessed the interaction of these clock genes polymorphisms with cardiovascular factors on the risk or progression to AD in cohort of well-defined SCD and MCI patients. The second strength is the very long mean follow-up time of 13 years. Moreover, non-converter patients had a longer 
follow-up time than patients who converted to AD. This is crucial information as follow-up time could influence rate of conversion to AD. The long follow-up time in our sample allows us to minimize the risk of classifying as stable subjects carrying an Alzheimer pathology who will convert later. Indeed, the present study suggests an association between ApoE genotype and Clock T3111C with different cardiovascular risk factors in a cohort of SCD and MCI patients. This interaction could influence the progression to $\mathrm{AD}$ in this group of patients. Understanding the mechanisms by which genetic and cardiovascular risk factors contribute to AD could inspire the development of new personalized therapeutic approaches for this disease.

Author Contributions: Conceptualization, V.B. and B.N.; methodology, V.B., S.M., B.N.; software, S.M.; validation, V.B., J.B., S.M., G.G., S.B., S.P., I.P., M.C., L.B., S.S., B.N.; formal analysis, V.B., S.M., J.B., S.B., I.P.; investigation, V.B., J.B., S.M., G.G., S.B., S.P., I.P., M.C., L.B., S.S., B.N.; resources, V.B., J.B., S.M., G.G., S.B., S.P., I.P., M.C., L.B., S.S., B.N.; data curation, V.B., J.B., S.M., G.G., S.B., S.P., I.P., M.C., C.F., L.B., S.S., B.N.; writing-original draft preparation, V.B., J.B., S.M., G.G.; writing-review and editing, V.B., S.M., G.G., S.S., B.N.; visualization, V.B., J.B., S.M., G.G., S.B., S.P., I.P., M.C., C.F., L.B., S.S., B.N.; supervision, V.B., S.S., B.N.; project administration, V.B., S.S., B.N.; funding acquisition, V.B., S.S. All authors have read and agreed to the published version of the manuscript.

Funding: This research was funded by Ministero della Salute and Regione Toscana (grants $n^{\circ}$ GR-2010-2316359-Longitudinal clinical-neuropsychological study of subjective memory complaints) and by Fondazione Cassa di Risparmio di Firenze (ECRF16-CONTRIBUTO 2015.0713, SORBI).

Conflicts of Interest: No authors report any conflicts of interest for this study.

\section{References}

1. Sperling, R.A.; Aisen, P.S.; Beckett, L.A.; Bennett, D.A.; Craft, S.; Fagan, A.M.; Iwatsubo, T.; Jack, C.R.; Kaye, J.; Montine, T.J.; et al. Toward defining the preclinical stages of Alzheimer's disease: Recommendations from the National Institute on Aging-Alzheimer's Association workgroups on diagnostic guidelines for Alzheimer's disease. Alzheimer's Dement. 2011, 7, 280-292.

2. Jessen, F.; Amariglio, R.E.; van Boxtel, M.; Breteler, M.; Ceccaldi, M.; Chételat, G.; Dubois, B.; Dufouil, C.; Ellis, K.A.; van der Flier, W.M.; et al. A conceptual framework for research on subjective cognitive decline in preclinical Alzheimer's disease. Alzheimer's Dement. 2014, 10, 844-852.

3. Albert, M.S.; DeKosky, S.T.; Dickson, D.; Dubois, B.; Feldman, H.H.; Fox, N.C.; Gamst, A.; Holtzman, D.M.; Jagust, W.J.; Petersen, R.C.; et al. The diagnosis of mild cognitive impairment due to Alzheimer's disease: Recommendations from the National Institute on Aging-Alzheimer's Association workgroups on diagnostic guidelines for Alzheimer's disease. Alzheimer's Dement. 2011, 7, 270-279. [CrossRef]

4. Bessi, V.; Mazzeo, S.; Padiglioni, S.; Piccini, C.; Nacmias, B.; Sorbi, S.; Bracco, L. From Subjective Cognitive Decline to Alzheimer's Disease: The Predictive Role of Neuropsychological Assessment, Personality Traits, and Cognitive Reserve. A 7-Year Follow-Up Study. J. Alzheimer's Dis. 2018, 63, 1523-1535.

5. Brodaty, H.; Heffernan, M.; Kochan, N.A.; Draper, B.; Trollor, J.N.; Reppermund, S.; Slavin, M.J.; Sachdev, P.S. Mild cognitive impairment in a community sample: The Sydney Memory and Ageing Study. Alzheimer's Dement. 2013, 9, 310-317.e1. [CrossRef]

6. Mitchell, A.J.; Beaumont, H.; Ferguson, D.; Yadegarfar, M.; Stubbs, B. Risk of dementia and mild cognitive impairment in older people with subjective memory complaints: Meta-analysis. Acta Psychiatr. Scand. 2014, 130, 439-451. [CrossRef]

7. Raichlen, D.A.; Bharadwaj, P.K.; Nguyen, L.A.; Franchetti, M.K.; Zigman, E.K.; Solorio, A.R.; Alexander, G.E. Effects of simultaneous cognitive and aerobic exercise training on dual-task walking performance in healthy older adults: Results from a pilot randomized controlled trial. BMC Geriatr. 2020, 20, 83. [CrossRef]

8. Reiter, K.; Nielson, K.A.; Smith, T.J.; Weiss, L.R.; Alfini, A.J.; Smith, J.C. Improved Cardiorespiratory Fitness Is Associated with Increased Cortical Thickness in Mild Cognitive Impairment. J. Int. Neuropsychol. Soc. JINS 2015, 21, 757-767.

9. World Health Organization. Risk Reduction of Cognitive Decline and Dementia: WHO Guidelines; WHO: Geneva, Switzerland, 2019; p. 401.

10. Norton, S.; Matthews, F.E.; Barnes, D.E.; Yaffe, K.; Brayne, C. Potential for primary prevention of Alzheimer's disease: An analysis of population-based data. Lancet Neurol. 2014, 13, 788-794. [CrossRef] 
11. Cazaly, E.; Charlesworth, J.; Dickinson, J.L.; Holloway, A.F. Genetic Determinants of Epigenetic Patterns: Providing Insight into Disease. Mol. Med. 2015, 21, 400-409. [CrossRef]

12. Ko, C.H.; Takahashi, J.S. Molecular components of the mammalian circadian clock. Hum. Mol. Genet. 2006, 15, R271-R277.

13. Hood, S.; Amir, S. Neurodegeneration and the Circadian Clock. Front. Aging Neurosci. 2017, 9, 170. [CrossRef]

14. Leng, Y.; Musiek, E.S.; Hu, K.; Cappuccio, F.P.; Yaffe, K. Association between circadian rhythms and neurodegenerative diseases. Lancet Neurol. 2019, 18, 307-318. [CrossRef]

15. Bonney, S.; Kominsky, D.; Brodsky, K.; Eltzschig, H.; Walker, L.; Eckle, T. Cardiac Per2 functions as novel link between fatty acid metabolism and myocardial inflammation during ischemia and reperfusion injury of the heart. PLoS ONE 2013, 8, e71493. [CrossRef]

16. Gómez-Abellán, P.; Hernández-Morante, J.J.; Luján, J.A.; Madrid, J.A.; Garaulet, M. Clock genes are implicated in the human metabolic syndrome. Int. J. Obes. 2008, 32, 121-128. [CrossRef]

17. Pagliai, G.; Sofi, F.; Dinu, M.; Sticchi, E.; Vannetti, F.; Molino Lova, R.; Ordovàs, J.M.; Gori, A.M.; Marcucci, R.; Giusti, B.; et al. CLOCK gene polymorphisms and quality of aging in a cohort of nonagenarians-The MUGELLO Study. Sci. Rep. 2019, 9, 1-7. [CrossRef]

18. McKhann, G.M.; Knopman, D.S.; Chertkow, H.; Hyman, B.T.; Jack, C.R.; Kawas, C.H.; Klunk, W.E.; Koroshetz, W.J.; Manly, J.J.; Mayeux, R.; et al. The diagnosis of dementia due to Alzheimer's disease: Recommendations from the National Institute on Aging-Alzheimer's Association workgroups on diagnostic guidelines for Alzheimer's disease. Alzheimer's Dement. 2011, 7, 263-269. [CrossRef]

19. Neary, D.; Snowden, J.S.; Gustafson, L.; Passant, U.; Stuss, D.; Black, S.; Freedman, M.; Kertesz, A.; Robert, P.H.; Albert, M.; et al. Frontotemporal lobar degeneration: A consensus on clinical diagnostic criteria. Neurology 1998, 51, 1546-1554.

20. Gorelick, P.B.; Scuteri, A.; Black, S.E.; Decarli, C.; Greenberg, S.M.; Iadecola, C.; Launer, L.J.; Laurent, S.; Lopez, O.L.; Nyenhuis, D.; et al. Vascular contributions to cognitive impairment and dementia: A statement for healthcare professionals from the american heart association/american stroke association. Stroke 2011, 42, 2672-2713. [CrossRef]

21. Bracco, L.; Amaducci, L.; Pedone, D.; Bino, G.; Lazzaro, M.P.; Carella, F.; D’Antona, R.; Gallato, R.; Denes, G. Italian Multicentre Study on Dementia (SMID): A neuropsychological test battery for assessing Alzheimer's disease. J. Psychiatr. Res. 1990, 24, 213-226. [CrossRef]

22. Caffarra, P.; Vezzadini, G.; Dieci, F.; Zonato, F.; Venneri, A. Rey-Osterrieth complex figure: Normative values in an Italian population sample. Neurol. Sci. 2002, 22, 443-447. [CrossRef]

23. Baddeley, A.; Della Sala, S.; Papagno, C.; Spinnler, H. Dual-task performance in dysexecutive and nondysexecutive patients with a frontal lesion. Neuropsychology 1997, 11, 187-194.

24. Spinnler, H.; Tognoni, G. Standardizzazione e Taratura Italian di Test Neuropsicologici: Gruppo Italiano per lo Studio Neuropsicologico Dell'invecchiamento; Masson Italia Periodici: Milano, Italy, 1987.

25. Giovagnoli, A.R.; Del Pesce, M.; Mascheroni, S.; Simoncelli, M.; Laiacona, M.; Capitani, E. Trail making test: Normative values from 287 normal adult controls. Ital. J. Neurol. Sci. 1996, 17, 305-309. [CrossRef]

26. Brazzelli, M.; Della Sala, S.; Laiacona, M. Taratura della versione italiana del Rivermead Behavioural Memory Test: Un test di valutazione ecologica della memoria. Giunti Organ. Spec. 1993, 206, 33-42.

27. Colombo, L.; Sartori, G.; Brivio, C. Stima del quoziente intellettivo tramite l'applicazione del TIB (Test Breve di Intelligenza). Giornale Italiano di Psicologia 2002, 29, 613-638.

28. Nelson, H. National Adult Reading Test (NART): For the Assessment of Premorbid Intelligence in Patients with Dementia: Test Manual; Windsor: Windsor, UK, 1982.

29. Hamilton, M. A rating scale for depression. J. Neurol. Neurosurg. Psychiatry 1960, 23, 56-62. [CrossRef]

30. Mishima, K.; Tozawa, T.; Satoh, K.; Saitoh, H.; Mishima, Y. The 3111T/C polymorphism of hClock is associated with evening preference and delayed sleep timing in a Japanese population sample. Am. J. Med Genet. Part B Neuropsychiatr. Genet. 2005, 133, 101-104.

31. Choub, A.; Mancuso, M.; Coppedè, F.; LoGerfo, A.; Orsucci, D.; Petrozzi, L.; DiCoscio, E.; Maestri, M.; Rocchi, A.; Bonanni, E.; et al. Clock T3111C and Per2 C111G SNPs do not influence circadian rhythmicity in healthy Italian population. Neurol. Sci. 2011, 32, 89-93. [CrossRef]

32. Saffroy, R.; Lafaye, G.; Desterke, C.; Ortiz-Tudela, E.; Amirouche, A.; Innominato, P.; Pham, P.; Benyamina, A.; Lemoine, A. Several clock genes polymorphisms are meaningful risk factors in the development and severity of cannabis addiction. Chronobiol. Int. 2019, 36, 122-134. [CrossRef] 
33. Becker-Krail, D.; McClung, C. Implications of circadian rhythm and stress in addiction vulnerability. F1000Research 2016, 5, 59.

34. Perreau-Lenz, S.; Spanagel, R. Clock genes $\times$ stress $\times$ reward interactions in alcohol and substance use disorders. Alcohol 2015, 49, 351-357. [CrossRef] [PubMed]

35. Bessi, V.; Giacomucci, G.; Mazzeo, S.; Bagnoli, S.; Padiglioni, S.; Balestrini, J.; Tomaiuolo, G.; Piaceri, I.; Carraro, M.; Bracco, L.; et al. Per2 C111G polymorphism influences cognition in Subjective Cognitive Decline and Mild Cognitive Impairment. A 10-year follow-up study. Eur. J. Neurol.. under review.

36. Bennet, A.M.; Di Angelantonio, E.; Ye, Z.; Wensley, F.; Dahlin, A.; Ahlbom, A.; Keavney, B.; Collins, R.; Wiman, B.; de Faire, U.; et al. Association of apolipoprotein E genotypes with lipid levels and coronary risk. JAMA 2007, 298, 1300-1311. [CrossRef] [PubMed]

37. Tang, G.; Wang, D.; Long, J.; Yang, F.; Si, L. Meta-analysis of the association between whole grain intake and coronary heart disease risk. Am. J. Cardiol. 2015, 115, 625-629. [CrossRef]

38. Gorelick, P.B. Risk factors for vascular dementia and Alzheimer disease. Stroke 2004, 35, 2620-2622. [CrossRef]

39. van der Flier, W.M.; Scheltens, P. Epidemiology and risk factors of dementia. J. Neurol. Neurosurg. Psychiatry 2005, 76, v2-v7. [CrossRef]

40. Rawle, M.J.; Davis, D.; Bendayan, R.; Wong, A.; Kuh, D.; Richards, M. Apolipoprotein-E (Apoe) $\varepsilon 4$ and cognitive decline over the adult life course. Transl. Psychiatry 2018, 8, 1-8. [CrossRef]

41. Xu, W.-L.; Caracciolo, B.; Wang, H.-X.; Santoni, G.; Winblad, B.; Fratiglioni, L. Accelerated progression from mild cognitive impairment to dementia among APOE $\varepsilon 4 \varepsilon 4$ carriers. J. Alzheimers Dis. JAD 2013, 33, 507-515. [CrossRef]

42. Hong, Y.J.; Yoon, B.; Shim, Y.S.; Kim, S.-O.; Kim, H.J.; Choi, S.H.; Jeong, J.H.; Yoon, S.J.; Yang, D.W.; Lee, J.-H. Predictors of Clinical Progression of Subjective Memory Impairment in Elderly Subjects: Data from the Clinical Research Centers for Dementia of South Korea (CREDOS). Dement. Geriatr. Cogn. Disord. 2015, 40, 158-165. [CrossRef]

43. Mazzeo, S.; Padiglioni, S.; Bagnoli, S.; Bracco, L.; Nacmias, B.; Sorbi, S.; Bessi, V. The dual role of cognitive reserve in subjective cognitive decline and mild cognitive impairment: A 7-year follow-up study. J. Neurol. 2019, 266, 487-497. [CrossRef]

44. Salameh, T.S.; Rhea, E.M.; Banks, W.A.; Hanson, A.J. Insulin resistance, dyslipidemia, and apolipoprotein E interactions as mechanisms in cognitive impairment and Alzheimer's disease. Exp. Biol. Med. 2016, 241, 1676-1683.

(C) 2020 by the authors. Licensee MDPI, Basel, Switzerland. This article is an open access article distributed under the terms and conditions of the Creative Commons Attribution (CC BY) license (http://creativecommons.org/licenses/by/4.0/). 